Angiologica 1970;7:382-384

\title{
Index rerum vol. 7
}

$\alpha$ - and B-blockers $147 \alpha$-napbtylacetate-esterase 1 Acetylcholine 129, 140 Adrenaline (Epinepbrine) 104, 147 Anatomy 160 splanchnic vessels 160 Aneurysm 28

aorta 28

dissecting 28

ectomy 246

localization 28

physical signs 28

survival data 28

symptom 28

treatment 28 Aneurysmectomy 246

aortic 246 Angiology 193

external 193

functional 193

general 193

internal 193

micro 193 Angiopathy 193

histangiopathy 193

micro- 193 Angiotensin 291, 338 Anisotropy of vessel wall 257 Aorta 28, 365

endothelial cell boundaries 365

scanning electron microscopy 365

silver nitrate staining 365 Aorta abdominalis 324

chondroitin-sulphate 325

collagen 324

dermatan-sulphate 325

elastin 324

glycogen 327

heparin 325

hcparin-sulphate 325

hexosamine 325

hyaluronate 325

lactate 327

lipids 324

mucopolysaccharides 324

oxygen-uptake 327

uronic acid 325 Arterial ischemia 187 Arterial metabolism 78, 327 Arterial wall 1

vasa vasorum 1 Arteriosclerosis 44, 312

antibody 44

biomicroscopical examination 312

childhood 44 Artery 8, 374

femoral 8

lesion of small arteries 374 Artificial thrombus 84 ATPaseactivity 273

denaturating agents 279

myosin 273

reducing agents 276

tonoactomyosin 273 Autoregulatory escape 336

ß- and /S-blockers 147 Blood-brain barrier 357

experimental thermal injury 357

permeability 357 Blood vessels 291

angiotensin 29

serotonin 291

testicular 291 Burn 357

Capillary endothelium 1 Cervical lymphatic blockage 25 Cholesterol embolism 246 Clonidine 296 Collagen 324 Coronary vessels 147 $\alpha$ - and /J-blockers 147

contractility 147

cow 147

electrical stimulation 147

epinephrine 147

histamin 147

horse 147

ligation 65,77 
Index rerum

383

Coronary potassium 147 serotonin 147

Dehydrogenase activity 109 Diabetes 348

serum content of $\beta$ - and pre-/5-lipoproteincholesterol 344 Diathermophoretic index 109 Dissecting aneurysm 28

Electrical stimulation 147 Electron microscopy 84

human platelets aggregates 84 Electrophoresis 346

agarose-agar gel 346 Essential hypertension 204

venous reflex activity 204 Estimation of serum 344

$\beta$ - and pre-/5-lipoproteins 344

jß-lipoproteín cholesterol 344 Euglobulinlysis time 20, 39

adrenalin 104

adrenergic receptor blocking agents 104

haemodialysis 20

noradrenalin 104

renal disease 20

shunt arterial blood 20

skin disease 39

Fibrinolytic activity 351 body weight 351 different races 351

Haemodialysis 20 Histamin 147

Histological conjunctival examination 312 Hydroxy-polyethoxydodecan 182 Hypertension 296 Hyperventilation 204

Idiopathic cramps 187 Infinitesimal theory 257 Intermittent claudicatio 212

arterial origin 212

non vascular origin 212 Intestinal blood flow 129

acetylcholine 129, 140

autoregulatory escape 140

in vitro perfusion 129

in vivo perfusion 140

intestinal musculature 129

intraluminal intestinal pressure 129 Ischemia 226

lower extremity 226

surgical therapy 226

Jugular vein ligation 53 fine structure of brain 53

Kidney circulation 333

Ligation of descending ramus of

coronary artery 65, 77 Lymphoedema 51

(/î-hydroxyethyl) rutosides 51, 187

experimental 51

treatment 51 Lymphogenous encephalopathy 57

lipid droplets 57

pericytes 57 Lymphostatic ophtalmus 25

panthotenic acid 25

pyridoxine 25

treatment 25

Method of measuring water 'avidity'

117 Mucopolysaccharides 324 Muscular work 8 Myocardial infarction 348

arterial and venous circulation 65

arterio-arterial anastomoses 65

arterio-venous anastomoses 65

experimental 65

proportion of blood flow/arterio-arterial, arterio-venous anastomoses 77

serum content of $\beta$ - and pre- $¿$ S-lipoproteincholesterol 348 Myosin (cow carotid) 273

ATPaseactivity

Noradrenaline 6, 104, 336

Obliterating arteriopathy 8 Occlusion 233

differential diagnosis 233

etiology 233

splanchnic vessels 160

vena cava 233

Phonocardiography 8

muscular work 8

obliterating arteriopathy 8

peripheral vascular hyperactivity 8 Plasminogen activator 177

blood content 177

vascular regions 177 Platelets aggregates 84 Potassium 147 Pressure distention 257

elastic cylinders 257

Raynaud 187 
primary 187 Renal artery embolization 246 Renal blood flow 335 Renal disease 20

euglobulinlysis time 20

384

Index rerum

Revascularization 226 tibial artery 226

Sclerosants 182

Hydroxy-polyethoxydodecan 182 Seborrheic alopecia 109 Semi-closed thromboendart

226 Serotonin 147 Skin disease 39 Skin flow 109

natrium ${ }^{\mathrm{BI}} \mathrm{J}$ clearance 109 Skin temperature 109 Spinal cord circulation 246 Splanchnic vessels 160

anatomy 160

clinical symptoms 160

occlusion 160 Systemic fibrosis 233

Tetradecylsulphate 182 Thorotrast 357 Tololazoline 296 Tonoactomyosin 273 ATPaseactivity 273 Triethyltin poisoning 117 Trimeresurus venom 84 Tryptic digestion 273 Turbidimetrie 344

Vasa vasorum 1

phosphatase reaction 1 representation 1

Vasalva maneuver 204

Vasoactive substances of vascular

smooth muscle 147 Vasoconstrictor fibres 337 Vasofilators 7 Vasomotor response 109 Vena cava 233

occlusion Vena cava inf. 321

chondroitin-sulphate 325

collagen 324

dermatan-sulphate 325

elastin 324

glycogen 327

heparin 325

heparin-sulphate 325

hexosamine 325

hyaluronate 325

lactate 327

lipids 324

mucopolysaccharides 324

oxygen-uptake 327

uronic acid 325 Veno-venous anastomoses 65, 77 Venomotor tone 204 Venoruton 51 Venous insufficiency 187

treatment Venous metabolism 327 Venous reflex 204

Xenon clearance 296 